\title{
Retrograde flow in the false lumen: Marker of a false lumen under stress?
}

Nicholas S. Burris, MD, ${ }^{\mathrm{a}}$ Himanshu J. Patel, MD, ${ }^{\mathrm{b}}$ and Michael D. Hope, MD, ${ }^{\mathrm{c}}$ Ann Arbor, Mich, and San Francisco, Calif

From the ${ }^{\mathrm{a}}$ Department of Radiology, University of Michigan, Ann Arbor, Mich; ${ }^{\mathrm{b}}$ Department of Cardiac Surgery, University of Michigan, Ann Arbor, Mich; and ${ }^{\mathrm{c}}$ Department of Radiology and Biomedical Imaging, University of California San Francisco, San Francisco, Calif.

Funded by NSB-Radiologic Society of North America Research Fellow Grant (RF1502).

Disclosures: Authors have nothing to disclose with regard to commercial support.

Received for publication April 13, 2018; revisions received June 25, 2018; accepted for publication June 28, 2018; available ahead of print Aug 18, 2018

Address for reprints: Nicholas S. Burris, MD, Department of Radiology, University of Michigan, 1500 E Medical

Center Dr, TC B1-132, SPC-5030, Ann Arbor, MI 48109-5030 (E-mail: nburris@med.umich.edu).

J Thorac Cardiovasc Surg 2019;157:488-91

$0022-5223 / \$ 36.00$

Copyright (c) 2018 by The American Association for Thoracic Surgery

https://doi.org/10.1016/j.jtcvs.2018.06.092

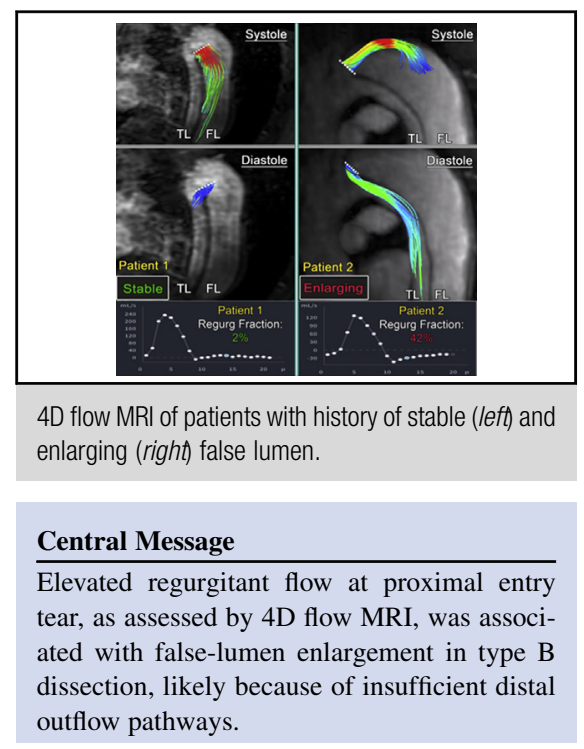

See Editorial Commentary page 492
Hemodynamic stress is thought to be a primary driver of progressive enlargement of the false lumen (FL) among patients with type B dissection. The proposed mechanism involves an imbalance between FL inflow and outflow pathways, leading to elevated FL pressures. Current risk stratification methods, however, rely on anatomic rather than hemodynamic characteristics (eg, maximal aortic diameter, entry tear size and location). Recent studies that have examined anatomic features on computed tomographic images to estimate FL outflow found that decreased FL outflow, but not maximal aortic diameter, predicts risk of future adverse events. ${ }^{1,2}$ An indirect estimation of FL outflow that is based on anatomy may not capture complex and variable blood flow in patients with dissection; however, direct measurement of flow in the FL may improve risk estimation. Increased retrograde FL flow has been shown to occur with decreased distal FL outflow pathways in studies that used computerized blood flow simulations, and these retrograde flow patterns can be easily identified by 3-dimensional phase-contrast (4D flow) magnetic resonance imaging (MRI). ${ }^{3,4} \mathrm{~A}$ simplified approach to assessing FL outflow obstruction, and thus elevated FL pressure, may therefore be to measure the degree of retrograde flow at the dominant entry tear, assuming that blood that cannot exit the FL distally will return to the true lumen during diastole.

\section{METHODS AND RESULTS}

Between November 2014 and December 2017, 12 patients with chronic dissection of the descending thoracic aorta $(n=9$ type $B ; n=3$ repaired type A) were prospectively enrolled in an Institutional Review Boardapproved study (HUM0000120679) and underwent contrast-enhanced 4D flow MRI with the goal of enrolling equal-sized groups of patients with enlarging and stable FL dimensions. Patients were excluded for the following reasons: (1) history of connective tissue disease, (2) complete FL thrombosis, and (3) no identifiable entry tear in the descending thoracic aorta. Entry tear regurgitant fraction was defined as the volume of retrograde flow returning to the true lumen during diastole over the entry tear forward systolic stoke volume. Electrocardiographically gated magnetic resonance angiography and computed tomographic angiography were used to define anatomic characteristics. Patients were defined as having an "enlarging" lesion if aortic growth rate was at least $3 \mathrm{~mm} / \mathrm{y}$, calculated by comparing maximal aortic diameter at baseline and on their most recent clinical computed tomographic angiographic examination. Continuous variables were examined for normality with the Shapiro-Wilk test. Those normally distributed were reported as mean $\pm \mathrm{SD}$ and those nonnormally distributed were reported as median and interquartile range. Stable and enlarging groups were compared with the Student $t$ test (parametric) or Mann-Whitney U test (nonparametric). Fisher exact tests were used to evaluate differences in frequency of categoric variables given small cell sizes. Correlation with aortic growth rate was assessed with the Spearman correlation.

Median patient age was 52 years (interquartile range, 48 -53 years), the majority were male (10/12), and the minority had partial FL thrombosis (3/12). The average age of the dissections was $3.7 \pm 3.1$ years. Half the patients had a history of recent FL enlargement (6/12 overall; 6/9 with type B and 0/3 with repaired type A). The dominant entry tear was located either at the distal arch/ proximal descending level $(\mathrm{n}=9)$ or mid descending level $(\mathrm{n}=3)$, and the mean dominant entry tear size was $13.3 \pm 6.8 \mathrm{~mm}$. Regurgitant flow fraction at the proximal entry tear was significantly higher among patients with history of FL enlargement $(45.8 \% \pm 32.1 \%$ vs $11.3 \% \pm 11.4 \% ; P=.03)$. There was 
a moderate association between aortic growth rate and regurgitant fraction at the proximal entry tear $(\rho=0.57 ; P=.05)$. There were no significant differences in age of the dissection; entry tear size and location; FL flow volume, velocity, and eccentricity; or baseline anatomic features between enlarging and stable groups. Comparison of demographic, anatomic and 4D flow MRI variables between stable and enlarging groups are shown in Table 1. Two representative cases are shown in Figure 1: a patient with large entry tear $(3.1 \mathrm{~cm})$ but low entry tear regurgitant fraction $(2 \%)$ and stable FL dimensions (Figure 1, left column, and Video 1), versus a patient with comparatively smaller entry tear $(1.4 \mathrm{~cm})$ but elevated entry tear regurgitant fraction $(42 \%)$ and progressive FL enlargement (Figure 1, right column, and Video 2).

\section{DISCUSSION}

We found entry tear regurgitant fraction to be significantly elevated among patients with a history of FL enlargement. These results lend support to a theory that was put forth in 2007 by the International Registry of Acute Aortic Dissection that reduced outflow in the setting of partial FL thrombosis leads to higher the FL pressures. ${ }^{5}$ Does the association between elevated regurgitant flow and FL enlargement simply represent a consequence of aneurysmal degeneration, rather than a cause? This seems unlikely, considering that studies with computerized blood flow simulation have shown that retrograde flow is a direct consequence of decreased FL outflow in nonaneurysmal models and that FL outflow estimates taken from baseline computed tomographic studies (eg, before aneurysm development) predict future events. ${ }^{1,}$

FL outflow only tells half of the hemodynamic story, and its direct measurement is nearly impossible because of the

TABLE 1. Demographic and 3-dimensional phase-contrast magnetic resonance imaging parameters

\begin{tabular}{|c|c|c|c|}
\hline Characteristics & Stable FL $(n=6)$ & Enlarging FL $(n=6)$ & $P$ value \\
\hline Patient age (y) & $51[45-52]^{*}$ & $53[51-54]^{*}$ & $.22 \dagger$ \\
\hline \multicolumn{4}{|l|}{ Dissection type } \\
\hline Residual dissection (repaired type A) & $3 / 3(100 \%)$ & $0 / 3(0 \%)$ & .09 \\
\hline Type B & $3 / 9(33 \%)$ & $6 / 9(67 \%)$ & \\
\hline $\operatorname{Sex}(M: F)$ & $5: 1$ & $5: 1$ & .77 \\
\hline Age of dissection (y) & $4.9 \pm 3.5$ & $2.4 \pm 2.3$ & .16 \\
\hline Aortic growth rate $(\mathrm{mm} / \mathrm{y})$ & $0.3[0-2.1]^{*}$ & $7.9[5.8-9.5]^{*}$ & $.003 \dagger$ \\
\hline Maximum aortic diameter (mm) & $39.5 \pm 3.8$ & $51.2 \pm 9.3$ & .02 \\
\hline \multicolumn{4}{|l|}{ Maximum aortic diameter location } \\
\hline Distal $\operatorname{arch}($ No. and \%) & $1(17 \%)$ & $3(50 \%)$ & $.44 \ddagger$ \\
\hline Proximal descending (No. and \%) & $4(66 \%)$ & $2(33 \%)$ & \\
\hline Mid descending (No. and \%) & $1(17 \%)$ & $1(17 \%)$ & \\
\hline \multicolumn{4}{|l|}{ FL } \\
\hline Net flow $\S(\mathrm{L} / \mathrm{min})$ & $1.5[0.5-4.2]^{*}$ & $0.8[0.4-2.0]^{*}$ & $.52 \dagger$ \\
\hline Peak velocity $\S(\mathrm{cm} / \mathrm{s})$ & $61.7 \pm 38.5$ & $51.3 \pm 21.0$ & .58 \\
\hline Eccentricity $\S, \|$ & $0.22 \pm 0.7$ & $0.20 \pm 0.8$ & .65 \\
\hline Regurgitant fraction $\S(\%)$ & $6.7[3.3-37.3]^{*}$ & $38.4[6.1-65.8]^{*}$ & $.26 \dagger$ \\
\hline Dominant entry tear size (mm) & $10.5[10.0-15.7]^{*}$ & $13.2[11.7-16.7]^{*}$ & $.52 \dagger$ \\
\hline \multicolumn{4}{|l|}{ Dominant entry tear jet } \\
\hline Net flow (L/min) & $0.7[0.4-2.8]^{*}$ & $0.4[0.1-0.8]^{*}$ & $.14 \dagger$ \\
\hline Peak velocity $(\mathrm{cm} / \mathrm{s})$ & $134.2 \pm 39.6$ & $96.5 \pm 26.3$ & .08 \\
\hline Regurgitant fraction $(\%)$ & $11.3 \pm 11.4$ & $45.8 \pm 32.1$ & .03 \\
\hline Intimal flap movement $(\mathrm{mm}) \Upsilon$ & $4.0 \pm 2.8$ & $1.5 \pm 1.4$ & .08 \\
\hline \multicolumn{4}{|l|}{ Baseline CT anatomic characteristics } \\
\hline FL diameter $(\mathrm{mm})$ & $23[20-25]^{*}$ & $21.5[16-24]^{*}$ & $.75 \dagger$ \\
\hline Entry tear distance from LSC (mm) & $15[2-65]^{*}$ & $27[20-127]^{*}$ & $.30^{\dagger}$ \\
\hline Inner curvature FL location (No. and \%) & $2(33 \%)$ & $2(33 \%)$ & .73 \\
\hline No. of secondary tears\# & $3.5 \pm 3.3$ & $2.6 \pm 1.7$ & 6 \\
\hline Secondary tear area $\left(\mathrm{mm}^{2}\right)^{* *}$ & $61[10-39]^{*}$ & $25[10-84]^{*}$ & $.37 \dagger$ \\
\hline Maximal aortic diameter $>40 \mathrm{~mm}$ ( No. and \%) & $2(33 \%)$ & $2(33 \%)$ & .73 \\
\hline FL diameter $>22 \mathrm{~mm}($ No. and $\%)$ & $4(67 \%)$ & $3(50 \%)$ & .50 \\
\hline \multicolumn{4}{|l|}{ Partial FL thrombus } \\
\hline Baseline CTA (No. and \%) & $2(33 \%)$ & $3(50 \%)$ & .5 \\
\hline Most recent CTA (No. and \%) & $2(33 \%)$ & $5(83 \%)$ & .24 \\
\hline
\end{tabular}

FL, False lumen; $C T$, computed tomography; $L S C$, left subclavian artery; $C T A$, computed tomographic angiography. *Median and interquartile range. $\dagger$ By Mann-Whitney $\mathrm{U}$ test. $\ddagger$ By $\chi^{2}$ test. $\S$ Measured $2 \mathrm{~cm}$ distal to dominant entry tear. ||Defined as normalized flow displacement. 9 Distance of intimal flap movement between peak-systole and end-diastole. \#Visible on oldest electrocardiographically gated computed tomographic angiography. **Assumes tear circularity. 

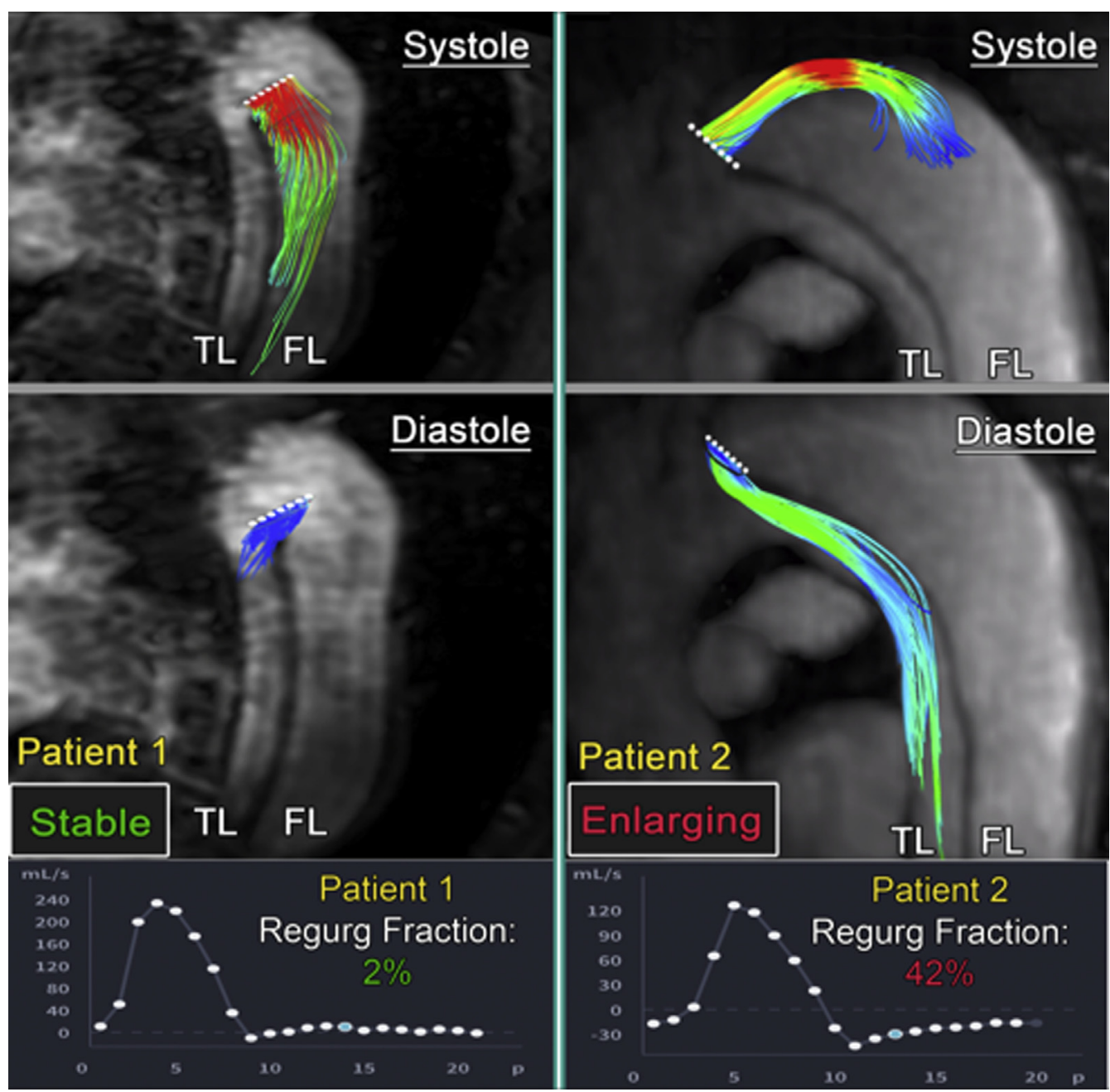

FIGURE 1. Three-dimensional phase-contrast magnetic resonance imaging of patients with history of stable (left) and enlarging (right) false lumen $(F L)$. $T L$, True lumen.

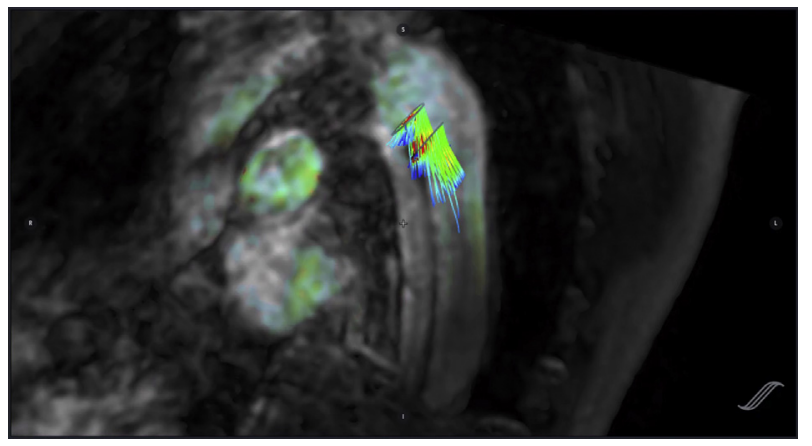

VIDEO 1. Sagittal 3-dimensional phase-contrast magnetic resonance imaging streamline visualization of a patient with type B aortic dissection and stable false-lumen dimensions. Regurgitant fraction at the proximal entry tear was low $(2 \%)$, and although the entry tear was large $(3.1 \mathrm{~cm})$, there was a similarly sized reentry tear at the level of the diaphragm. Video available at: https://www.jtcvs.org/article/S0022-5223(18)32024-5/fulltext. often numerous and small outflow pathways. Alternatively, direct measurement of entry tear regurgitant faction with 4D flow MRI is an intuitively appropriate approach aimed at quantifying the FL hemodynamic stress that occurs as a result of insufficient FL outflow pathways. Our study is limited by its small size, 4D flow MRI's inability to directly measure FL pressure, and the fact that FL growth status was known at the time of patient enrollment. Although our results require further validation in larger studies, we believe that an imaging approach that uses direct FL blood flow measurements in addition to anatomic factors may significantly improve currently available risk assessment techniques. In light of the increasing rates of endovascular repair among patients with type B dissection, 4D flow MRI assessment of FL blood flow may improve patient risk assessment and allow prophylactic repair to be targeted to patients with the highest degree of hemodynamic stress. 


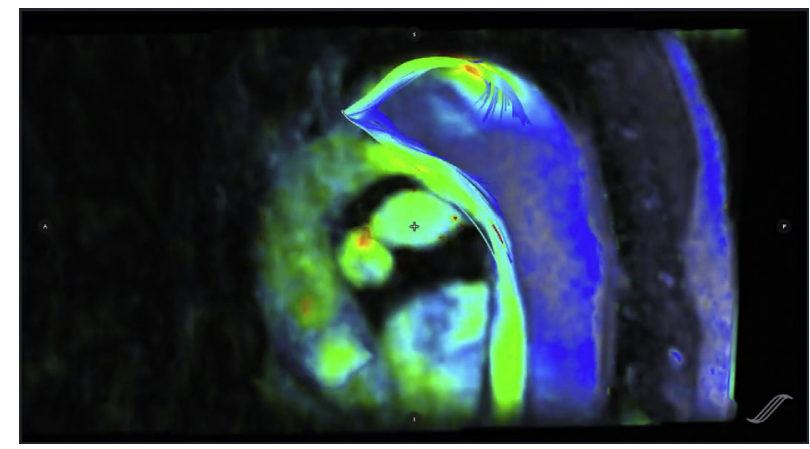

VIDEO 2. Sagittal 3-dimensional phase-contrast magnetic resonance imaging streamline visualization of a patient with type B aortic dissection and progressively enlarging false-lumen dimensions. Regurgitant fraction at the proximal entry tear was significantly elevated $(42 \%)$, and there were not large distal reentry tears identified on computed tomography. Video available at: https://www.jtcvs.org/article/S0022-5223(18)32024-5/fulltext.

\section{References}

1. Sailer AM, van Kuijk SM, Nelemans PJ, Chin AS, Kino A, Huininga M, et al Computed tomography imaging features in acute uncomplicated Stanford type-B aortic dissection predict late adverse events. Circ Cardiovasc Imaging. 2017;10:e005709.

2. Sailer AM, Nelemans PJ, Hastie TJ, Chin AS, Huininga M, Chiu P, et al. Prognostic significance of early aortic remodeling in acute uncomplicated type B aortic dissection and intramural hematoma. J Thorac Cardiovasc Surg. 2017;154:1192-200.

3. Rudenick PA, Segers P, Pineda V, Cuellar H, García-Dorado D, Evangelista A et al. False lumen flow patterns and their relation with morphological and biomechanical characteristics of chronic aortic dissections. Computational model compared with magnetic resonance imaging measurements. PLoS One. 2017;12: $\mathrm{e} 0170888$.

4. François CJ, Markl M, Schiebler ML, Niespodzany E, Landgraf BR, Schlensak C et al. Four-dimensional, flow-sensitive magnetic resonance imaging of blood flow patterns in thoracic aortic dissections. J Thorac Cardiovasc Surg. 2013;145: 1359-66.

5. Tsai TT, Evangelista A, Nienaber CA, Myrmel T, Meinhardt G, Cooper JV, et al International registry of acute aortic dissection. Partial thrombosis of the false lumen in patients with acute type B aortic dissection. N Engl J Med. 2007;357: 349-59. 